\title{
Evaluación preliminar de la Estrategia de Información, Comunicación y Educación para el control del Aedes aegypti, en La Dorada, Colombia
}

Evaluating an Information, Education and Communication (IEC) strategy which was adopted for Aedes aegypti control in La Dorada, Colombia

Adriana del Pilar Pacheco-Coral ${ }^{1}$, Marta L. Quiñones-Pinzón ${ }^{1}$, Idalba M. Serrato-Pomar ${ }^{2}$ y Favio A. Rivas-Muñoz ${ }^{1}$

1 Facultad de Medicina, Universidad Nacional de Colombia. Bogotá. adpachecoc@unal.edu.co, mlquinonesp@unal.edu.co, farivasm@unal.edu.co

2 Facultad de Ciencias, Universidad del Tolima. Ibagué, Colombia.mildrepora@yahoo.es

Recibido 1 Octubre 2009/Enviado para Modificación 23 Mayo 2010/Aceptado 26 Junio 2010

\section{RESUMEN}

Objetivo Estimar el impacto de la estrategia de Información, Educación y Comunicación sobre la infestación de Aedes aegypti en viviendas de La Dorada. Métodos Estudio transversal adelantado entre junio y julio de 2008 en La Dorada, Colombia. Se seleccionaron 228 viviendas donde se midió presencia del vector a través del método rapid sweeping para formas inmaduras en tanques y de captura activa para adultos. Simultáneamente, una encuesta midió la exposición a la estrategia. La base de datos se analizó en EPI info 2000. Se calcularon Razones de Prevalencia e intervalos de confianza al $95 \%$.

Resultados Las viviendas que no tenían larvas se caracterizaban por tener mujeres cabeza de familia o moradores con conocimiento acerca de las larvas y la forma de transmisión del dengue. Aquellas viviendas donde se lavaba el tanque de agua al menos una vez por semana, carecían de formas inmaduras. La presencia de mosquitos adultos era más frecuente en las casas que tenían más de un tanque. Cerca del $80 \%$ de los encuestados adquirieron el conocimiento de transmisión del dengue a través de la estrategia.

Conclusiones Si bien la estrategia no ha sido aplicada en todos sus componentes, se encontraron factores que podrían tener un efecto protectivo al estar relacionados con ausencia del vector. El estudio revela solo un éxito parcial de la estrategia en La Dorada.

Palabras Clave: Aedes, dengue, entomología (fuente: DeCS, BIREME).

ABSTRACT

Objective Estimating the impact of the Information, Education and Communication strategy on Aedes aegypti infestation in homes in La Dorada, Colombia. 
Methods A cross-sectional study was carried out during June and July 2008 in La Dorada; 228 houses were chosen and vector presence was measured in two ways: immature forms were captured by the rapid sweeping method in water tanks and adults were captured by active collection. A survey simultaneously measured exposure to the strategy. EPI Info 2000 was used for analysing the database so created. Prevalence ratios and $95 \%$ confidence intervals were calculated.

Results A relationship was found between being a housewife and not having larvae present in the home. No immature forms were found in houses where somebody washed the water tank at least once a week. There were also no larvae in homes where people had knowledge about larvae and dengue transmission. The presence of adult forms was found in houses having more than one water tank. Almost $80 \%$ of the interviewees acquired knowledge about dengue transmission through the strategy.

Conclusions Although all strategy components had not been implemented, several factors were found which might have a protective effect (i.e. being related to vector absence). The study showed that the strategy achieved only partial success in La Dorada.

Key Words: Aedes aegypti, dengue, entomology (source: MeSH, NLM).

$\mathrm{E}$ n la actualidad, el dengue es la enfermedad viral transmitida por vectores más relevante por su morbilidad y mortalidad, ya que anualmente se presentan 10 millones de casos y 20000 muertes en el mundo (1). En Colombia, la tasa de dengue clásico para 2006 fue de147,7/100 000 hab., y para 2007 de 183/100 000 hab (2). Hasta hoy no se dispone de tratamiento específico ni de vacuna, por tanto, la mayoría de las acciones en prevención y control en el mundo se han dirigido al vector. La iniciativa mundial para el control del dengue se estableció en 1995 e incluía el control integrado e intersectorial del vector (el cual abarca el uso tanto de los diferentes tipos de control convencionales como también de la participación comunitaria), el saneamiento ambiental, la urbanización planificada y el uso correcto de los insecticidas (3). El control integrado contempla acciones que involucran la comunidad en procesos de comunicación de información y de movilización social, tal como lo plantean las estrategias Información, Educación y Comunicación (IEC) y, Comunicación para el Impacto Conductual (COMBI) (4). En consonancia con las políticas mundiales, a finales de los noventa, el Ministerio de Salud incorporó acciones de control integral e intersectorial del vector y estrategias de participación comunitaria (5). Es así como el país cuenta con experiencias descentralizadas, en municipios y departamentos, de participación comunitaria. Actualmente, el Plan Nacional de Salud Pública (6) incluye al dengue dentro de las prioridades en salud y estas estrategias se contemplan en los Planes Territoriales de Salud. Con estos antecedentes no debe perderse de vista que entre los aspectos que permiten la 
sostenibilidad en el tiempo y el adecuado cumplimiento de los objetivos propuestos dentro de la estrategia IEC, se encuentran la evaluación continua de sus procesos y los resultados, así como también el monitoreo y la retroalimentación a la comunidad (7). Sin embargo, es muy poca la información publicada en materia de evaluación.

En uno de los municipios endémicos del departamento de Caldas, La Dorada, se ha llevado a cabo la estrategia IEC como parte del programa de prevención y control municipal de dengue que se ha desarrollado desde 1981. La Dorada cuenta, hasta el momento, con indicadores obtenidos anualmente que han permitido medir el cumplimiento de los objetivos y los resultados alcanzados para evaluar los procesos. El desarrollo local de la estrategia IEC tiene como pilares: 1. la capacitación de personas claves como vigías de la salud, estudiantes de último grado de educación secundaria, promotoras de la salud e individuos pertenecientes a organizaciones gubernamentales y no gubernamentales que repliquen conocimientos sobre la enfermedad, su prevención, el vector y su control; 2. la comunicación a través de prensa, radio, televisión y folletos y; 3 . las jornadas de recolección de inservibles, en las que confluyen la población, los líderes comunitarios y sectores diferentes al de la salud (8).

El propósito de este estudio fue evaluar y contrastar los componentes de la estrategia IEC implementada en La Dorada frente a la presencia o ausencia de mosquitos vectores en las viviendas.

\section{MATERIALES Y MÉTODOS}

Diseño del estudio

Se realizó un estudio transversal durante el período comprendido entre Mayo y Noviembre de 2008.

Área de estudio

Municipio de La Dorada, ubicado a una altura de $178 \mathrm{msnm}$., con temperatura promedio de $27^{\circ} \mathrm{C}$, y una humedad relativa del 75 al $79 \%$. Se escogieron los barrios Las Ferias y Las Margaritas.

Sujetos de estudio

El estudio incluyó a 228 personas cabeza de hogar que fueran mayores de edad, vivieran y permanecieran en sus viviendas. 
Muestra y muestreo

Se utilizó un muestreo por conglomerados en aquellos barrios donde se reportó el mayor número de casos en años anteriores, la mayor densidad de mosquitos y donde la estrategia IEC se había implementado (9). Dentro de los barrios, se seleccionaron de forma aleatoria 10 manzanas en las que se visitó semanalmente el $25 \%$ de las viviendas. En ellas se aplicó una encuesta a la persona cabeza de hogar.

Prueba piloto

Se llevó a cabo en el mes de abril de 2008 una prueba en ambos barrios. A partir de ésta se pudo establecer si la encuesta tenía un diseño adecuado y también si la forma de medir la presencia del vector era efectiva.

Obtención de información acerca de la estrategia IEC

Se obtuvo información de las acciones desarrolladas por la Secretaría Municipal de Salud, Educación y Bienestar Social durante el 2007 y comienzos del 2008.

Mediciones entomológicas y epidemiológicas

La medición del vector (en términos de presencia o ausencia) se realizó en el momento del ingresó a la vivienda. Se clasificaron los depósitos positivos para la presencia de larvas y pupas. Así mismo, se solicitó la información del número de habitantes de la vivienda y se realizó la colecta de ejemplares de Ae.aegypti en sus diferentes estadios. La recolección de estadios inmaduros consistió en tomar una muestra de agua realizando 3 barridos con una red de acuario, metodología adaptada de Romero-Vivas et. al (10). Por otra parte, la captura de mosquitos adultos se basó en búsqueda activa de los mismos durante 10 minutos con una red entomológica (11). Para la recolección de datos epidemiológicos se aplicaron 228 encuestas. Cada encuesta tenía seis secciones en las que se preguntaba acerca de aspectos sociales y económicos, alcances de la estrategia IEC según el criterio de las personas, conocimientos frente a la enfermedad y al vector, prácticas y actitudes de autocuidado.

Análisis de datos

Los datos se analizaron en el programa EPI INFO 2000. Las variables de interés fueron descritas. El análisis bivariado se hizo calculando razones de prevalencia e intervalos de confianza del $95 \%$. Se realizó análisis estratificado para revisar la existencia de las posibles asociaciones libres de confusión. 


\section{RESULTADOS}

Características demográficas

Se incluyeron 228 individuos en el estudio. El promedio de edad fue 42,5 años y la razón mujeres a hombres fue de 5:1. Todos los individuos incluidos pertenecían al estrato socioeconómico 1 . Cerca del $86 \%$ de los participantes tenía algún grado de escolaridad.

Percepción de la estrategia IEC

De acuerdo con la respuesta de los participantes, la frecuencia de visitas domiciliarias de promotoras fue de 3,5\% y la de estudiantes fue de 3,9\% durante el año previo a la realización de las visitas. La difusión de información a través de medios de comunicación, convencionales y no convencionales mostró que el $86 \%$ de la población no había recibido folletos informativos y que menos del $50 \%$ de los conocimientos habían sido adquiridos por propagandas de televisión o radio. Los conocimientos que las personas tenían sobre la enfermedad y el vector habían sido adquiridos, en más del $50 \%$ de los casos, por otras vías que no incluían ningún componente de la estrategia. La distribución de los medios, a través de los cuales la población adquirió los conocimientos, se muestra en la Tabla 1.

Tabla 1. Medios de adquisición de conocimientos de dengue

\begin{tabular}{lcccc}
\hline \multirow{2}{*}{ Respuesta conocimiento } & \multicolumn{4}{c}{ Medio de adquisición del conocimiento } \\
\cline { 2 - 5 } & $\begin{array}{c}\text { Visitas } \\
\%\end{array}$ & $\begin{array}{c}\text { Capacitaciones } \\
\%\end{array}$ & $\begin{array}{c}\text { Propagandas } \\
\%\end{array}$ & $\begin{array}{c}\text { Otros } \\
\%\end{array}$ \\
\hline Dengue & 16 & 17 & 19 & 51 \\
Transmisión dengue & 10 & 30 & 36 & 25 \\
Zancudo transmisor & 17 & 59 & 12 & 12 \\
Zancudo vive dentro de vivienda & 2 & 12 & 5 & 80 \\
"Saltones" & 1 & 8 & 7 & 84 \\
"Saltón" vive en agua limpia & 4 & 8 & 4 & 85 \\
"Saltón" vive dentro de la vivienda & 3 & 11 & 7 & 79 \\
\hline
\end{tabular}

Tabla 2. Conocimientos frente al dengue y al vector

\begin{tabular}{lccc}
\hline \multirow{2}{*}{ Tema } & \multicolumn{3}{c}{ Respuesta conocimiento } \\
\cline { 2 - 4 } & $\mathrm{Si}$ & No & Otra \\
& $\%$ & $\%$ & respuesta \% \\
\hline Dengue & 11 & 88 & 1 \\
Transmisión dengue & 78 & 22 & 0 \\
Zancudo transmisor & 7 & 93 & 0 \\
Zancudo vive dentro de vivienda & 45 & 31 & 34 \\
"Saltones" & 94 & 6 & 0 \\
Agua limpia o limpia estancada & 70 & 25 & 5 \\
"Saltón" vive dentro de la vivienda & 90 & 7 & 3 \\
\hline
\end{tabular}


Conocimientos sobre la enfermedad y el vector

De acuerdo a los resultados encontrados, las personas tenían más arraigados los conocimientos sobre el saltón (la larva del vector) y la transmisión de la enfermedad. La frecuencia del conocimiento de las personas, respecto al vector y a la enfermedad se ve en la Tabla 2.

Inspección visual de las viviendas

De las 228 viviendas, el 97,4 \% tenían alberca en el patio. El 2,2\% tenían tanque bajo adicional a la alberca y, el 25,3\% tenían un tanque alto. En el $63,8 \%$ de las viviendas se encontró una serie de diferentes objetos que constituían como depósitos artificiales de agua.

Tabla 3. Factores asociados con la presencia del vector Aedes aegypti:

\begin{tabular}{lccccc}
\multicolumn{1}{c}{ actitudes y prácticas } & & \multicolumn{2}{c}{ IC 95\% } \\
\multicolumn{1}{c}{ Factor } & $\begin{array}{c}\text { Presencia de Ae. } \\
\text { aegypti }\end{array}$ & N & R P & Mínimo & Máximo \\
\hline Cabeza de familia mujer & Larvas & 228 & 0,69 & 0,49 & 0,99 \\
$\begin{array}{l}\text { Lavado de la alberca 1 a 4 } \\
\text { veces por semana }\end{array}$ & Pupas & 222 & 0,49 & 0,28 & 0,87 \\
$\begin{array}{l}\text { Lavo la alberca dentro de los 7 } \\
\text { días previos }\end{array}$ & Pupas & 225 & 0,44 & 0,26 & 0,76 \\
$\begin{array}{l}\text { Lavo la alberca dentro de los 7 } \\
\text { dias previos a la visita }\end{array}$ & Larvas & 225 & 0,57 & 0,42 & 0,78 \\
Existencia de tanque bajo & Piel de pupas & 228 & 3,43 & 1,11 & 10,65 \\
\hline
\end{tabular}

Análisis bivariado

Se encontraron diversos factores relacionados con la presencia o no del vector dentro de las viviendas, entre los que se encontraron ser mujer cabeza de familia $(\mathrm{RP}=0,69$, IC95 \%:0,49-0,99), conocimientos tales como la definición de saltón ( $\mathrm{RP}=0,62$, IC95 $\%: 0,39-0,98)$, el saltón se cría dentro de la vivienda $(\mathrm{RP}=0,67$, IC95 \%:0,45-0,99) $y$ forma de transmisión del dengue $(\mathrm{RP}=0,71, \mathrm{IC} 95 \%: 0,51-0,99)$, prácticas como lavado de la alberca dentro de la semana previa a la visita y entre 1 y 4 veces por semana y presencia de tanque bajo adicional a la alberca (Tablas 3 y 4).

Tabla 4. Factores asociados con la presencia del vector Aedes aegypti: conocimientos

\begin{tabular}{lccccc}
\hline \multicolumn{1}{c}{ Factor } & $\begin{array}{c}\text { Presencia de } \\
\text { Ae. aegypti }\end{array}$ & N & R P & \multicolumn{2}{c}{ IC 95\% } \\
& Larvas & 228 & 0,71 & 0,51 & 0,99 \\
\hline $\begin{array}{l}\text { Conocimiento "forma de } \\
\text { transmisión dengue" }\end{array}$ & Depósito positivo & 221 & 0,67 & 0,45 & 0,99 \\
$\begin{array}{l}\text { Conocimiento "saltones viven } \\
\text { dentro de vivienda" }\end{array}$ & Depósito positivo & 221 & 0,67 & 0,45 & 0,99 \\
$\begin{array}{l}\text { Conocimiento "saltones viven } \\
\text { dentro de vivienda" }\end{array}$ & Larvas & 228 & 0,62 & 0,39 & 0,98 \\
$\begin{array}{l}\text { Conocimiento "saltones" } \\
\text { Conocimiento "saltones" }\end{array}$ & Piel de pupas & 228 & 0,36 & 0,15 & 0,89 \\
\hline
\end{tabular}


Tabla 5. Análisis estratificado

\begin{tabular}{|c|c|c|c|c|}
\hline Factor & $\begin{array}{c}\text { Presencia de } A e . \\
\text { aegypti }\end{array}$ & $\begin{array}{c}\text { Estratificado por la } \\
\text { variable }\end{array}$ & $\begin{array}{l}\text { RP Cruda } \\
\text { (IC } 95 \% \text { ) }\end{array}$ & $\begin{array}{l}\text { RP ajustada } \\
\text { (IC } 95 \% \text { ) }\end{array}$ \\
\hline $\begin{array}{l}\text { Existencia } \\
\text { de tanque bajo }\end{array}$ & Piel de pupas & Con. "saltones" & $\begin{array}{c}3,43 \\
(1,11-10,65)\end{array}$ & $\begin{array}{c}2,34 \\
(0,73-7,56)\end{array}$ \\
\hline $\begin{array}{l}\text { Conocimiento } \\
\text { "saltones" }\end{array}$ & Piel de pupas & $\begin{array}{l}\text { Existencia de } \\
\text { tanque bajo }\end{array}$ & $\begin{array}{c}0,36 \\
(0,15-0,89)\end{array}$ & $\begin{array}{c}0,45 \\
(0,17-1,13)\end{array}$ \\
\hline
\end{tabular}

Análisis estratificado

Al realizar la estratificación, solamente las variables existencia de tanque bajo y conocimiento del término saltón mostraron disminución de la razón de prevalencias al realizar el ajuste. A pesar de que el intervalo de confianza dejó de ser significativo, la relación entre el factor estudiado y la infestación por el vector no cambió (Tabla 5).

\section{DISCUSIÓN}

Esta evaluación de la estrategia IEC en el municipio de La Dorada permitió encontrar factores, como algunos conocimientos y prácticas, relacionados de alguna manera con la infestación intradomiciliaria por Ae. aegypti y evidenciar si los componentes de la estrategia, estaban siendo efectivos en la transmisión de los mensajes y de la información sobre dengue. El hecho de que la mujer sea ama de casa se asocio con la no presencia del vector dentro del hogar. Esta observación también se ha encontrado en otras investigaciones (12-15). Otros autores han hallado que la mujer tiende a realizar la práctica de la limpieza doméstica mientras que el hombre tiende a participar en la recolección de basuras $(16,17)$. Por tanto, el rol de ama de casa podría explicar la disminución de criaderos. Según Pozo et al la actividad de limpieza que realiza el ama de casa, puede conllevar a prácticas como el lavado frecuente de la alberca. Esta es una de las prácticas que se promueven a través de la estrategia IEC en este municipio y se encontró relacionada con disminución de la presencia de larvas y pupas si se realizaba dentro de los 7 días previos a la visita y con una frecuencia de entre 1 y 4 veces a la semana. Por otra parte, que la vivienda tuviera tanque bajo y alberca se relacionó con tener piel de pupa, el cual es un indicador indirecto de eclosión de mosquitos adultos $(18,19)$. La relación entre este factor e infestación continuó intacta a pesar de la estratificación por otras variables. Esto ratifica que los tanques bajos sin tapa o con tapa deteriorada continúan siendo uno de los principales criaderos del mosquito tal como lo refieren algunos autores cubanos, peruanos y colombianos (20-22). Aunque alrededor del $90 \%$ de las viviendas en La Dorada cuentan con servicio de acueducto, lo que garantizaría un suministro ininterrumpido de agua potable, los pobladores continúan almacenando este recurso en tanques (23). A pesar que la estrategia IEC en el municipio intenta informar 
acerca del tapado correcto y disposición de tanques, estos resultados muestran que al parecer este esfuerzo no es suficiente para que las personas realicen esta práctica. A este respecto, Periago y Guzmán sugieren que se requeriría de la participación activa de las personas para que ellas dieran la importancia a esta práctica y la pusieran en marcha. Frente a los conocimientos, la mayor parte de las personas desconocían la sintomatología del dengue. Autores colombianos han descrito que en regiones endémicas del país las personas confunden este cuadro con el de gripa común (24-26). El desconocimiento de esta enfermedad conlleva a que las personas no le den relevancia suficiente como problema de salud, lo que dificulta su prevención. Sin embargo, el conocimiento frente a la forma de transmisión de la enfermedad si fue evidente y además se relacionó con no tener infestación por larvas. Los pobladores refirieron que ese conocimiento fue adquirido principalmente en propagandas transmitidas a través de los medios de comunicación. A este respecto, varios autores han encontrado que el uso de los medios de comunicación influye en la adquisición de conocimientos sobre dengue en la población expuesta a los mismos $(27,28)$. Sin embargo el conocimiento que se encontró arraigado en los individuos fue el del saltón (larva) y que éste se encontraba dentro de la vivienda. Hubo relación entre tener los dos conocimientos y no tener larvas. Así mismo entre el segundo y no tener la alberca positiva para alguna forma del vector. La mayoría de los individuos obtuvo estos conocimientos por otros medios diferentes a la estrategia, lo que sugiere que los pobladores tienen vías alternas de comunicación para difundir estos conocimientos y que son efectivas para la realización de prácticas que disminuyen la infestación de Ae. aegypti en las albercas. La relación entre saber acerca del saltón y no tener piel de pupas no varió notablemente en el análisis estratificado, aunque dejó de ser estadísticamente significativo.

El diseño de este estudio tiene tanto ventajas como limitaciones. De estas últimas, cabe anotar que la medición simultánea de la estrategia IEC y las entomológicas no permiten establecer temporalidad y, por tanto, atribuir causalidad. Además, no permite conocer los cambios en el tiempo que haya ocasionado la estrategia. Respecto a la posible introducción de errores sistemáticos se intentó evitarlos a través de las siguientes acciones: 1. No fueron reemplazadas las personas que se negaron a participar dentro del estudio para obviar el sesgo de selección; 2. El instrumento con el que se midió la exposición se diseñó a partir de lo encontrado en la literatura científica y se validó en prueba piloto. Algunas de las preguntas tienen un criterio de verificación para tratar de realizar una adecuada clasificación. Es posible que se haya presentado un sesgo de mala clasificación entre los expuestos y los no expuestos, por fallas en el instrumento, pero sin diferencia entre los sujetos de estudio, lo cual llevaría a una subestimación de la 
Razón de Prevalencia y, por tanto, la asociación pudiera ser más fuerte. Por otro lado, algunas de las respuestas de los individuos pudieron influir sobre su clasificación, dado que ellos no respondieron o dieron respuestas incompletas. Es importante aclarar que, en el momento de realizar la encuesta los entrevistadores desconocían cuales eran los resultados arrojados por las mediciones entomológicas. También que el método para obtener estas últimas está validado en el ámbito científico y quienes realizaron la medición fueron entrenados por personal experimentado $(29,30)$.

Finalmente, la importancia de este estudio radica en que permitió explorar la exposición a la estrategia, a través de sus diferentes frentes de acción, y las mediciones del vector en sus diferentes estadios. También la posibilidad de generar hipótesis de investigación y la identificación de posibles factores asociados al evento que puedan ser estudiados de manera más amplia posteriormente (31). La escogencia de esas zonas de La Dorada, se hizo teniendo en cuenta que allí se han venido presentado la mayoría de los casos de dengue en el municipio así como las encuestas aédicas más altas. Esto conlleva a que la estrategia se ejecute allí, quizá con más empeño que en otras regiones del municipio. Por consiguiente, se espera que estos datos permitan a las autoridades de salud fortalecer los componentes de la estrategia con miras a lograr un control efectivo e integral del vector y en esa medida, contribuir a la disminución de los casos de dengue en esas zonas del municipio *

Agradecimientos: A la Dirección Territorial de Salud de Caldas, a la Alcaldía de La Dorada y a la población de La Dorada por la colaboración prestada para la realización de este trabajo. A la Universidad Nacional de Colombia por la financiación de este estudio.

\section{REFERENCIAS}

1. Dengue Fever Home Page Centers for Disease Control Division of vector-borne infectious disease [Internet]. Disponible en: www.cdc.gov.co. Consultado en 2008.

2. Instituto Nacional De Salud. Sistema de Vigilancia en Salud Pública [Internet]. Disponible en: www.ins.gov.co. Consultado Abril de 2008.

3. Periago MR, Guzmán MG. Dengue y dengue hemorrágico en las Américas. Rev Panam Salud Publica 2007; 21(4):187-191.

4. Parks W, Lloyd L. Planificación de la movilización y comunicación social para la prevención y el control del dengue: guía paso a paso. Ginebra: Organización Mundial de la Salud; 2004.

5. Instituto Nacional de Salud, Grupo de vigilancia en Salud Pública. Prevención y control del dengue. Informe ejecutivo semanal. Bogotá: Instituto Nacional de Salud; 2001.

6. República de Colombia Ministerio de la Protección Social. Decreto 3039 de 2007. "Por el cual se adopta el Plan Nacional de Salud Pública 2007-2010". Bogotá: Ministerio de la Protección Social; 2007. 
7. World Health Organization. Information, education and communication. Switzerland; 2001.

8. Alcaldía de La Dorada. Desarrollo de las estrategias IEC, Información, Educación y Comunicación, para las enfermedades de interés en salud pública y campañas de promoción y prevención año 2007 [Internet]. Disponible en: http://www.ladorada-caldas.gov.co. Consultado Marzo de 2008.

9. Dirección territorial de salud de Caldas. Boletín epidemiológico semanal de Caldas, semana 52. Caldas: Dirección territorial de salud de Caldas; 2007.

10. Romero C, Llina H, Falconar AK. Three Calibration Factors, Applied to a Rapid Sweeping Method, Can Accurately Estimate Aedes aegypti (Diptera: Culicidae) Pupal Numbers in Large Water-Storage Containers at All Temperatures at Which Dengue Virus Transmission Occurs. J Med Entomol 2007; 44(6):930-937.

11. Castro M, Quintana N, Quiñones M. Evaluación de dos piretroides en el control del vector del dengue en Putumayo, Colombia. Rev Salud Publica 2007; 9(1):106-116.

12. Kusriastuti R, Suroso T, Nalim S, Kusumadi W. "Together Picket": community activities in dengue source reduction in Purwokerto City, Central Java, Indonesia. Dengue Bull WHO 2004; 28(Suppl):35-38.

13. Méndez J, Rivas L. Dengue prevention in Mérida, Yucatán, Mexico: use of formative research to refine an education/communication intervention targeting household management of key Aedes aegypti-producing containers. Dengue Bull WHO 2004; 28(Suppl): 44-47.

14. Pozo EJ, Neira M, Vilchez E, Melendez M. Factores asociados a la infestación por Aedes aegypti en el distrito de Tambogrande, Piura 2004. Rev Peru Med Exp 2007;24(2):144-151.

15. Sinh V, Kay B, Thi N, Ryan P, Bek A. Community mobilization, behaviour change and biological control in the prevention and control of dengue fever in Viet Nam. Dengue Bull WHO 2004; 28(Suppl): $57-61$.

16. Pérez CL, Seda H, García EJ, Clark GG. Knowledge and attitudes in Puerto Rico concerning dengue prevention. Rev Panam Salud Publica 2005; 17(4):243-253.

17. Pérez CL, Zielinski E, Vargas D, Clark GG. Community beliefs and practices about dengue in Puerto Rico. Rev Panam Salud Publica 2009; 25(3):218-226.

18. Focks D. A Review of Entomological Sampling Methods and Indicators for Dengue Vectors. Switzerland: World Health Organization on behalf of the Special Programme for Research and Training in Tropical Diseases; 2003.

19. Rios JF. Aspectos entomológicos del dengue. Infectio 2004; 8(3):231-235.

20. Bisset JA, Marquetti MC, Portillo R, Rodríguez MM, Suárez S, Leyva M. Factores ecológicos asociados con la presencia de larvas de A. aegypti en zonas de alta infestación del municipio Playa, Ciudad de La Habana, Cuba. Rev Panam Salud Publica 2006; 19(6):379384.

21. Fernández W, lannacone J, Rodríguez E, Salazar N, Valderrama B, Morales AM, et al. Distribución espacial, efecto estacional y tipo de recipiente más común en los índices entomológicos larvarios de Aedes aegypti en Yurimaguas. Perú 2000-2004. Rev Peru Med Exp Salud Publica 2005; 22(3):191-199.

22. Tinker M, Olano V. Ecología del Aedes aegypti en un pueblo de Colombia, Sur América. Biomédica 1993; 13(1):5-14.

23. Departamento Administrativo Nacional De Estadística Boletín Censo General 2005, Perfil CaldasLa Dorada. [Internet]. Disponible en: http://www.dane.gov.co/files/cens 02005/perfiles/ caldas/la_dorada.pdf. Consultado Agosto del 2008.

24. Quintero J, Carrasquilla G, Suarez R, Gonzalez C, Olano V. An ecosystemic approach to evaluating ecological, socioeconomic and group dynamics affecting the prevalence of Aedes aegypti in two Colombian towns. Cad. Saúde Pública 2009; (25):S93-S103.

25. Fajardo P, Monje CA, Lozano G, Realpe O, Hernández LE. Nociones populares sobre "dengue" y "rompehuesos", dos modelos de la enfermedad en Colombia Rev Panam Salud Publica 2001; 10(3):161-69. 
26. Suarez R, Olarte MF, González AC. Is what I have just a cold or is it dengue? Addressing the gap between the politics of dengue control and daily life in Villavicencio-Colombia. Soc Sci Med 2005; 61:495-502.

27. Wing K, Nang S, Min A. Community/based assessment of dengue/related knowledge among caregivers. Dengue Bull 2004; 28:189-195.

28. Ávila GA, Martínez M, Sherman C, Fernández E. Evaluación de un módulo escolar sobre dengue y Aedes aegypti dirigido a escolares en Honduras. Rev Panam Salud Publica. 2004; 16(2):84-94.

29. Kleinbaum D, Sullivan K, Barker N. How to Set Things Up? Study Designs. In: A Pocket Guide to Epidemiology. New York: Springer Science+Business Media, LLC; 2007.

30. Rivas F. Epidemiología: Fundamentos. Bogotá: Editorial Facultad de Medicina Universidad Nacional de Colombia; 2008

31. Hernández B, Velasco-Mondragón H. Encuestas transversales. Salud Pública Mex 2000; 42(5):447-455. 